\title{
BMJ Open Relieving acute pain (RAP) study: a proof-of-concept protocol for a randomised, double-blind, placebo- controlled trial
}

\author{
Luana Colloca, ${ }^{1}$ Se Eun Lee, ${ }^{1}$ Meghan Nichole Luhowy, ${ }^{2}$ Nathaniel Haycock, ${ }^{1}$ \\ Chika Okusogu, ${ }^{1}$ Soojin Yim, ${ }^{1}$ Nandini Raghuraman, ${ }^{1}$ Robert Goodfellow, ${ }^{3}$ \\ Robert Scott Murray, ${ }^{3}$ Patricia Casper, ${ }^{3}$ Myounghee Lee, ${ }^{4}$ Thomas Scalea, ${ }^{3}$ \\ Yvette Fouche, ${ }^{5}$ Sarah Murthi ${ }^{3}$
}

To cite: Colloca L, Lee SE, Luhowy MN, et al. Relieving acute pain (RAP) study: a proof-of-concept protocol for a randomised double-blind, placebocontrolled trial. BMJ Open 2019;9:e030623. doi:10.1136/ bmjopen-2019-030623

- Prepublication history for this paper is available online. To view these files, please visit the journal online (http://dx.doi org/10.1136/bmjopen-2019030623).

Received 17 July 2019 Revised 26 September 2019 Accepted 17 October 2019
Check for updates

(C) Author(s) (or their employer(s)) 2019. Re-use permitted under CC BY-NC. No commercial re-use. See rights and permissions. Published by BMJ.

For numbered affiliations see end of article.

Correspondence to Professor Luana Colloca; colloca@umaryland.edu

\section{ABSTRACT}

Introduction Physicians and other prescribing clinicians use opioids as the primary method of pain management after traumatic injury, despite growing recognition of the major risks associated with usage for chronic pain. Placebos given after repeated administration of active treatments can acquire medication-like effects based on learning mechanisms. This study hypothesises that dose-extending placebos can be an effective treatment in relieving clinical acute pain in trauma patients who take opioids.

Methods and analysis The relieving acute pain is a proofof-concept randomised, placebo-controlled, double-blinded, single-site study enrolling 159 participants aged from 18 to 65 years with one or more traumatic injuries treated with opioids. Participants will be randomly assigned to three different arms. Arm 1 will receive the full dose of opioids with non-steroidal anti-inflammatory drugs (NSAIDs). Arm 2 will receive the $50 \%$ overall reduction in opioid dosage, dose-extending placebos and NSAIDs. Arm 3 (control) will receive NSAIDs and placebos. The trial length will be 3 days of hospitalisation (phase I) and 2-week, 1-month, 3-month and 6-month follow-ups (exploratory phase II). Primary and secondary outcomes include feasibility and acceptability of the study. Pain intensity, functional pain, emotional distress, rates of rescue therapy requests and patient-initiated medication denials will be collected.

Ethics and dissemination All activities associated with this protocol are conducted in full compliance with the Institutional Review Board policies and federal regulations. Publishing this study protocol will enable researchers and funding bodies to stay up to date in their fields by providing exposure to research activity that may not otherwise be widely publicised.

Date and protocol version identifier 3/6/2019 (HP00078742).

Trial registration number NCT03426137.

\section{INTRODUCTION}

Pain is a significant and costly public health problem in the USA. The economic burden of chronic pain and related morbidities cost the USA approximately $\$ 645$ billion for the

\section{Strengths and limitations of this study}

- This is the first study to assess feasibility and acceptability of dose-extending placebos to reduce opioid use in acute trauma pain both the short-term and long-term.

- The convenience of an app-based tool for data collection provides easy access for long-term follow-ups.

- Excluded populations, such as pregnant and breastfeeding women and prisoners, may affect the study's generalisability.

year 2012. ${ }^{1}$ According to the 2012 National Health Interview Survey, almost 50 million adults in the USA reported having significant, chronic or severe pain. ${ }^{2}$ Opioids are frequently prescribed for the management of any type of pain despite the lack of high-quality evidence demonstrating efficacy, effectiveness and safety of long-term opioid therapy for the management of chronic non-cancer pain. ${ }^{34}$ Opioids can induce drug tolerance (and the need for escalating doses), hyperalgesia (increased pain sensitivity) and addiction. ${ }^{5}$ Furthermore, the economic cost of opioid overdose, abuse and dependence was estimated to be $\$ 78.5$ billion for the year 2013. ${ }^{7}$ The opioid crisis is a public health disaster resulting in 16 deaths per day in the USA. ${ }^{89}$ More than a third of all opioidrelated overdoses can be attributed to prescription opioids. ${ }^{10}$ One out of 550 chronic opioid users dies within approximately 2.5 years of their first opioid prescription that is given to treat acute pain. ${ }^{11}$

One plausible strategy to fight against the opioid epidemic is to include placebos with analgesics for pain management. ${ }^{12} 13$ Extensive research on placebo analgesia over the past several decades has expanded knowledge 


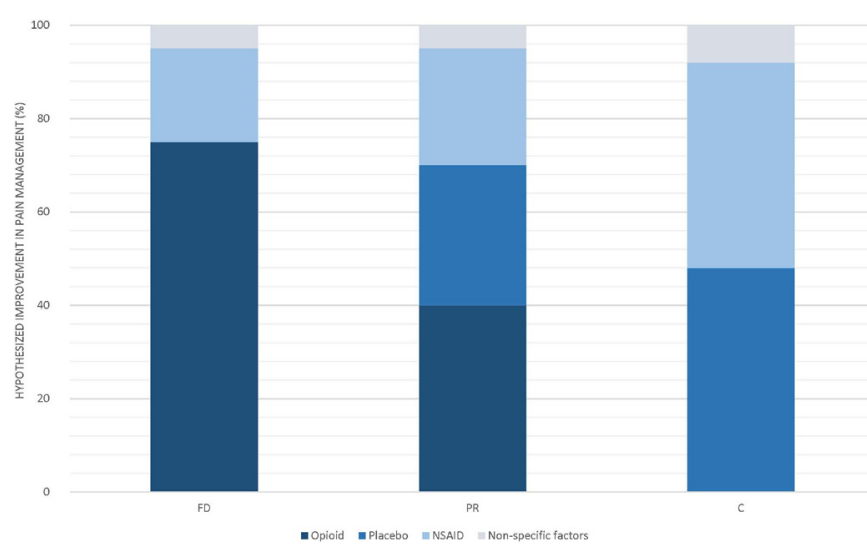

Figure 1 Hypothesised effect of various treatments on improvement in pain management for FD, PR and C treatment groups. FD, full dose group; PR, partial reduction group; C, control group. We hypothesise that all three groups' outcomes will improve, but that the three study drugs will contribute to this improvement to different extents.

of a fascinating psycho-neurobiological phenomenon underlying endogenous pain reduction. ${ }^{14}$ This provocative line of research involves the use of placebos to enhance therapeutic outcomes through learning paradigms that produce behavioural and biological responses mirroring those induced by active drugs. Studies indicate that placebos given after repeated administration of active treatments (eg, morphine) acquire a drug-like effect (eg, pain reduction) in both animals and humans. ${ }^{13}$ It has been well established that the effect of this modality is greater than that obtainable using placebos alone. ${ }^{16-19}$ Dose-extending placebos can remediate the associated challenges in pain management by triggering the endogenous pain modulatory systems in the body ${ }^{13}$ and there is a growing evidence for the effectiveness of dose-extending placebos and/or subclinical doses of pain treatments when they are blended with active treatments. ${ }^{20}$

We designed a proof-of-concept (PoC) study to test the feasibility and acceptability of a method that exploits learning mechanisms, via dose-extending placebos, to reduce opioid intake in traumatic pain. The study will also test how the reduction of opioids during hospitalisation will affect future use of opioids within a 6-month follow-up period. This novel prospect of placebo use could improve knowledge about how expectancies can modulate pain, as well as illuminate potential avenues to prevent chronic opioid use by reducing opioid treatments for those with acute pain.

\section{Objectives}

The primary objective is to establish an alternative method of reducing opioids. Dose-extending placebos could reduce overall opioid intake, decreasing physiological dependence, and still provide effective pain management, thus preventing pain interference with daily functioning. Participants will be enrolled from the R. Adams Cowley Shock Trauma Center (STC) in Baltimore Maryland. They will be randomised to three arms: Arm 1 will be a full dose (FD) group, which will receive oxycodone $(5 \mathrm{mg})$ and non-steroidal anti-inflammatory drugs (NSAIDs) dosed in accordance with the shock trauma guidelines for acute pain in Orthopaedic Injury. Arm 2 will be a partial reduction (PR) group, which will receive oxycodone $(5 \mathrm{mg})$, NSAIDs, and dose-extending placebos to reach a $50 \%$ reduction of the total intake of opioids. Finally, Arm 3 will be a control (C) group receiving NSAIDs and placebos. The local STC therapeutic protocol for pain management for NSAIDs includes ketorolac tromethamine intravenous (15 or $30 \mathrm{mg}$ every 8 hours), adjusted with ibuprofen, meloxicam and celecoxib based on patients' effectiveness and side effects profiles. Secondary objectives are to assess the acceptability and feasibility of recruiting participants with traumatic injuries to successfully participate in the trial, including the 2-week, 1-month, 3-month and 6-month follow-ups for pain and opioid use, are investigated. This PoC study will inform and determine appropriate power for a future larger randomised controlled trial.

\section{Study hypotheses}

H1: We hypothesise that participants in the PR arm will experience a clinically meaningful improvement in pain management as compared with the FD arm, as detailed in figure 1 .

H2: We hypothesise that participants in the PR arm will experience significantly better outcomes than participants in the $\mathrm{C}$ arm.

H3: We hypothesise feasibility and acceptability of this method of reducing opioids on adequate training of the clinical staff and family members.

\section{METHODS AND ANALYSIS \\ Study design}

We plan to conduct a double-blinded, randomised controlled PoC study to examine the learning mechanisms and efficacy of dose-extending placebos in participants with any traumatic injury requiring opioid medications for pain management. We will recruit and randomise 159 participants with traumatic injuries to one of three study arms according to a 1:1:1 schedule of randomisation: arm 1/FD group will receive oxycodone $(5 \mathrm{mg})$ and NSAIDs and will be dosed in accordance with the STC therapeutic protocol for pain management guidelines for NSAIDs. Arm 2/PR group will receive oxycodone $(5 \mathrm{mg})$, NSAIDs and placebos to reach a $50 \%$ reduction of the total intake of opioids. Finally, arm 3/control (C) group will receive NSAIDs and placebos. The study schema is presented in figure 2 .

\section{Setting}

The study will be conducted at the STC, a free-standing dedicated trauma hospital and provides the highest level of care for critically ill and injured participants in the state as the primary adult resource centre for Maryland's emergency medical services system. The STC is the specialty referral centre for the State of Maryland for neurotrauma 


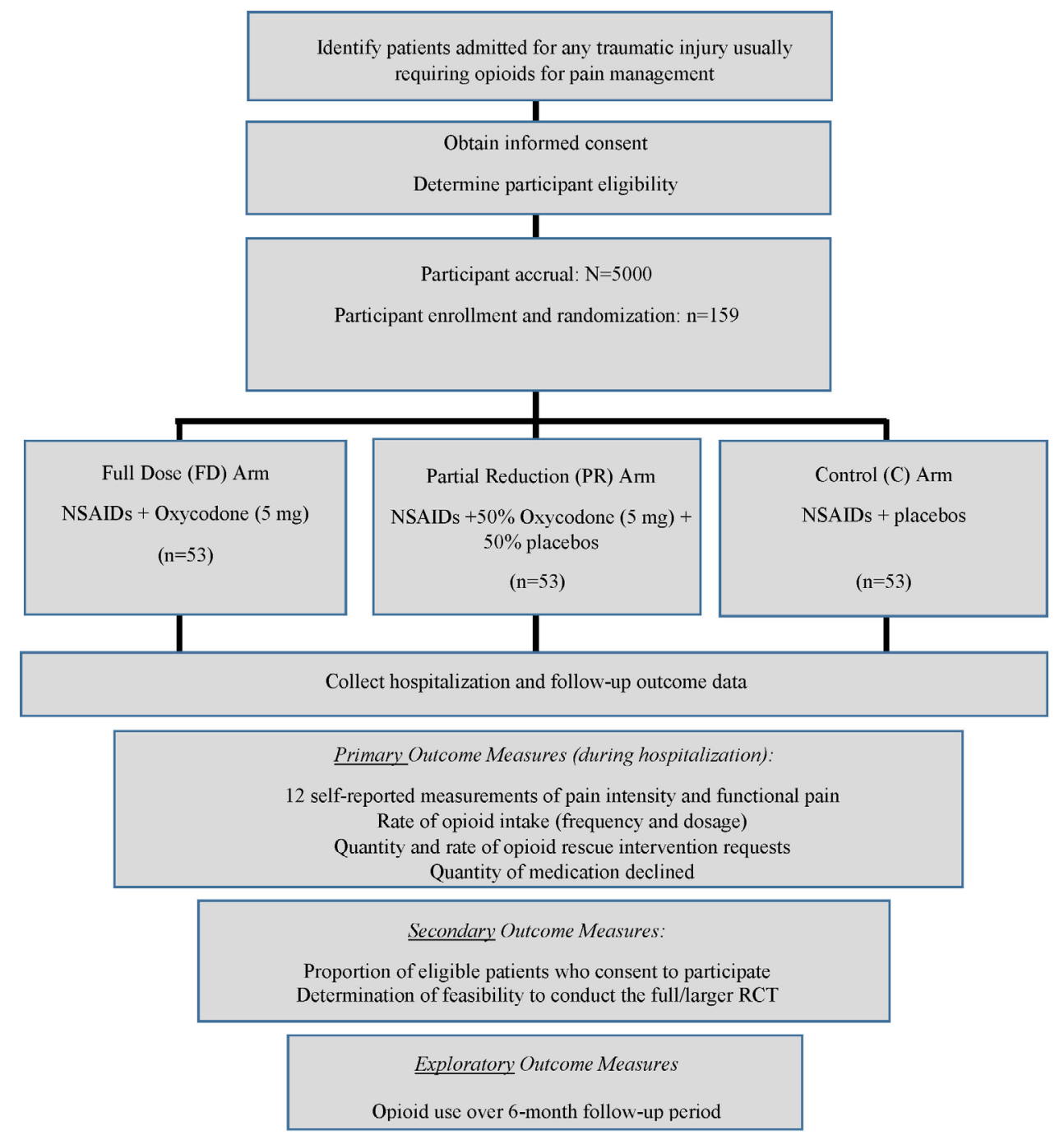

Figure 2 Study schema.

and orthopaedic surgery. This study will start recruiting in January 2020.

\section{Participant recruitment}

A total of 159 participants will be enrolled into this study. Participants will be identified by the study investigators' routine review of patients who have been admitted to the STC with a traumatic injury requiring opioid medication for pain management. The study investigator or research team delegate will approach potentially eligible patients to confirm interest in the study. If the patient is interested, the research team will discuss the study details as outlined in the Institutional Review Board (IRB)-approved informed consent form (ICF). Patients will be given ample time to read the ICF and ask questions. If the patient agrees to participate in the relieving acute pain trial, the patient and the research team member who conducted the informed consent discussion will sign and date the ICF and Health Insurance Portability and Accountability Act (HIPAA) authorisation form. The informed consent process is documented on a checklist which includes confirmation that the participant freely agrees to participate and comprehends the study purpose, procedures and expectations. The research team will proceed with confirmation of patient eligibility via chart review. Moreover, a plan by the primary service to adhere to the shock trauma protocol for NSAID use is a requirement for enrolment. Participants are free to withdraw their participation at any point in the study with or without reporting a reason. Withdrawal of participation in the study is also documented on a participation withdrawal form.

\section{Eligibility}

Broad eligibility is proposed to increase the generalizability and feasibility of the trial. There are no exclusions based on sex, race or ethnicity in this trial.

\section{Inclusion criteria}

In order to be eligible for participation in this study, participants must satisfy all following criteria: (1) 18-65year old, English-speaking adults, (2) admission to the STC within 72 hours of any traumatic injuries requiring inpatient opioid medication, (3) expected hospital stay $\geq 48$ hours, (4) consent to the care plan by primary service to follow the local shock trauma guidelines for acute pain in orthopaedic injury. 


\section{Exclusion criteria}

Patients will not be eligible to participate in the study if they satisfy any of the following criteria: (1) non-Englishspeaking adults, (2) spinal cord injury, (3) severe traumatic brain injury as per physician discretion, using Glasgow Coma Scale, (4) patient-controlled analgesia, (5) admission creatinine $>1.4$, (6) history of chronic kidney disease, (7) current active cancer being treated with opioid medications, (8) heroin use in the 3 months prior to admission (based on patient self-report), (9) severe psychiatric condition (eg, schizophrenia, bipolar disorders, mania, autism) and/or psychiatric condition leading to treatment and/or hospitalisation within the last 1 year, (10) positive toxicology screen for methadone not prescribed during current hospitalisation (including self-report of heroin in the past 3 months), (11) pregnancy or breast feeding, (12) contraindication to NSAIDs, including high risk of bleeding or known severe coronary artery disease, (13) injuries deemed non-survival, (14) discretion of investigators over eligibility of participants in situations where their injuries interfere with accurate pain ratings and participation would not adversely affect their well-being or recovery.

Currently, there is not a tool for screening those at risks of opioids misuses for trauma patients. ${ }^{21}$ Nevertheless, before the randomisation and study allocation we will screen participants using an adapted version of the opioid risk tool ${ }^{22}$ excluding those with a score of 8 or higher which indicates a high risk for opioid abuse.

\section{Randomisation and treatment allocation}

Participant randomisation will only occur once written informed consent is obtained and eligibility is confirmed by investigators. The study team will inform Investigational Drug Services (IDS) Pharmacy at the University of Maryland Medical Center of all newly enrolled participants in the study. IDS will then randomise participants according to the randomisation table generated by IDS pharmacy into one of the three treatment groups: NSAIDs with $5 \mathrm{mg}$ oxycodone (FD arm), NSAIDs with oxycodone and placebos (PR arm) or NSAIDs with placebo (C arm), according to a 1:1:1 schedule of randomisation. IDS pharmacy will be responsible for medication dispensing and delivery to the floor during the trial. The participant will start to receive the study drug within 24 hours of the normally planned opioid administration, ideally as the participant's first opioid medication while hospitalised. All staff involved with the protocol are fully trained on the procedures and are given emergency contacts of the study team in case of incident.

\section{Blinding}

Participants, investigators, care providers, data collectors and other research team members will not be aware of group allocation. The IDS pharmacy staff, who will prepare the blinded medications for each participant, will be unblind to the treatment assignment.

\section{Placebo and oxycodone suspension}

Oxycodone and placebo oral suspensions are manufactured by IDS Pharmacy at the University of Maryland Medical Center. All study medications are prepared as suspensions to accommodate administration with a potentially broad range of participants (eg, intubated participant). Oxycodone and placebo suspensions are identical in terms of appearance, taste and smell; both suspensions appear pink in colour and mint taste. Orasweet syrup vehicle is used as a flavouring vehicle for both oxycodone and placebo oral suspensions. IDS will determine the order of administration and delivers drug twice per day to be stored in a dedicated automated drug dispensing system for study participants. The pharmacy dispenses medications for each participant that corresponds with his/her allocated treatment group. Participants in the PR group receive four doses of oxycodone (5 mg) followed by one of four repeating schedules of administration alternating between oxycodone (5 mg) and placebo.

\section{Rescue therapy and unblinding}

All participants will receive $0.4-1 \mathrm{mg}$ of intravenous dilaudid or $1-4 \mathrm{mg}$ dilaudid by mouth every 1 hour as needed for rescue and break through pain. Adjuncts (eg, lidocaine patches and other medication) can be given as rescue therapy. The rescue therapy will be managed by the acute pain management service (APMS) and the team will appropriately document use of dilaudid rescue therapy and any additional administered medication. APMS will monitor participants for safety during hospitalisation (phase I). When participants are pending discharge, APMS will contact IDS for the study allocation to prescribe the adequate treatment. Based on pain severity interference, emotional distress and drug assignment during hospitalisation (phase I), participants who did not receive opioids as part of the research study randomisation will be discharged with NSAIDs avoiding the prescription of opioids. Participants will be instructed to take the prescribed therapies such as baclofen, cyclobenzaprine, lidocaine patches, NSAIDs, acetaminophen, gabapentin or pregabalin. Based on the severity of the trauma, a prescription of oxycodone $5 \mathrm{mg}$ every 4 hours, oxycodone $5 \mathrm{mg}$ every 6 hours or Oxycodone $5 \mathrm{mg}$ every 6 hours may be given for no more than 3-5 days and participants will be instructed to limit the opioids intake to strictly necessary circumstances. This is in accordance with current clinical practice at STC.

Thus, there are two instances in which the study team will become unblinded to treatment allocation: if a participant is being withdrawn from the study because they required more than three doses of rescue medication in a row, or at the end of phase I when APMS will recommend appropriate treatment for pain management. Otherwise, randomisation and treatment allocation will only be revealed to the study team once recruitment and data collection are complete. 
Table 1 Schedule of study assessments

\begin{tabular}{|c|c|c|c|c|c|c|c|c|c|c|}
\hline \multirow[b]{2}{*}{ Study event } & \multicolumn{5}{|c|}{ Phase I: hospitalisation } & \multicolumn{5}{|c|}{ Phase II: exploratory follow-up period* } \\
\hline & $\begin{array}{l}\text { Pre-study } \\
\text { period }\end{array}$ & SD1† & SD2 & SD3 & $\mathrm{D} / \mathrm{C} \neq$ & $\begin{array}{l}\text { 2-week } \\
\text { post } D / C\end{array}$ & $\begin{array}{l}\text { 1-month } \\
\text { post } D / C\end{array}$ & $\begin{array}{l}\text { 3-month } \\
\text { post } D / C\end{array}$ & $\begin{array}{l}\text { 6-month } \\
\text { post } D / C\end{array}$ & $\begin{array}{l}\text { Study } \\
\text { exit }\end{array}$ \\
\hline Screening and informed consent & $x$ & & & & & & & & & \\
\hline Randomisation & & $\mathrm{x}$ & & & & & & & & \\
\hline Health and medication use CRF & & $x \S$ & & & & $x$ & $\mathrm{X}$ & $\mathrm{X}$ & $\mathrm{X}$ & \\
\hline Study medication administration & & $\mathbf{x}$ & $x \emptyset$ & $x \emptyset$ & & & & & & \\
\hline \multicolumn{11}{|l|}{ Clinical assessments } \\
\hline PEG Questionnaire & & $X^{\star *}$ & $X^{\star \star}$ & $X^{* *}$ & & $x$ & $x$ & $x$ & $x$ & \\
\hline FPQ Questionnaire & & $X^{\star \star}$ & $X^{\star *}$ & $X^{\star *}$ & & $\mathrm{X}$ & $x$ & $\mathrm{X}$ & $\mathrm{X}$ & \\
\hline PCS Questionnaire & & $X^{\star *}$ & $X^{\star *}$ & $X^{\star \star}$ & & $x$ & $x$ & $x$ & $x$ & \\
\hline PDQ Questionnaire & & $X^{\star *}$ & $X^{\star \star}$ & $X^{* *}$ & & $x$ & $x$ & $x$ & $\mathrm{x}$ & \\
\hline $\begin{array}{l}\text { PROMIS Short Forms: } \\
\text { Pain Interference } 8 \mathrm{a}\end{array}$ & & $X^{\star \star}$ & $X^{\star \star}$ & $X^{\star \star}$ & & & & $x$ & $x$ & \\
\hline Pain Behaviour 7a & & $X^{\star \star}$ & $X^{\star *}$ & $X^{\star \star}$ & & & & $x$ & $\mathrm{x}$ & \\
\hline $\begin{array}{l}\text { Prescription Pain Medication } \\
\text { Misuse } 7 a\end{array}$ & & $X^{\star \star}$ & $X^{\star \star}$ & $X^{\star \star}$ & & & & $x$ & $x$ & \\
\hline Sleep Disturbance 8a & & $X^{* *}$ & $X^{* *}$ & $X^{\star *}$ & & & & $x$ & $x$ & \\
\hline Depression 8a & & $X^{* *}$ & $X^{\star \star}$ & $X^{* *}$ & & & & $x$ & $x$ & \\
\hline Anxiety $8 a$ & & $X^{\star *}$ & $X^{\star *}$ & $X^{\star \star}$ & & & & $\mathrm{x}$ & $x$ & \\
\hline Exit interview & & & & & & & & & & $\mathrm{X}$ \\
\hline
\end{tabular}

Patient-Reported Outcomes Measurement Information System (PROMIS) short-form scales are included in Table 1.

*All Phase II study events occur remotely (eg, research team communicates with participants via phone, participants complete questionnaires within secure app-based platforms).

†SD1, study day 1: occurs within 72 hours of hospitalisation when participants are administered the first study medication.

$\neq \mathrm{D} / \mathrm{C}$, discharge.

§Baseline health and medication use CRF.

IStudy medication administration occurs every 3 hours, for a maximum of 3 days hospitalised.

${ }^{* *}$ Questionnaire administered within 20-90 min following study medication administration.

CRF, case report form; FPQ, Fear of Pain Questionnaire; ICD-11, International Classification of Diseases 11th Revision; PCS, Pain Catastrophizing

Scale; PDQ, Pain Detect Questionnaire; PEG, pain intensity enjoyment of life and general activity.

\section{Hospitalisation}

Hospitalisation window (2-3 days hospitalisation): pain ratings will be recorded within 20-90 min after each study drug administration. Also, participants will be given the option to complete a psychological battery (see table 1) during the time in the hospital or by the time of their first follow-up visit on paper, over the phone or electronically via MetricWire, a secure HIPAA-compliant tool.

\section{Discharge}

Participants will be monitored for use of opioids at the first post-discharge visit at approximately 2 weeks, and at months 1,3 and 6 for follow-ups. The research team may communicate with participants via calls, letters, emails or texts. MetricWire will be used to facilitate communication and to promote participant retention. The team member will ask the participant questions about their health and medication use. In addition, a phone application may be developed or the data collection systems may be used to gather information about participants' medication use and health during the follow-up period.

\section{Phase I (hospitalisation)}

The hospitalisation period will be inclusive of study enrolment to participant discharge as shown in table 1.

\section{Laboratory testing and toxicology screens}

Participants are given an option to provide a blood sample while hospitalised and at the 6-month follow-up. Blood samples will be stored for future studies that will assess the correlation between circulating gene expression patterns and psychological and physiological phenotypes.

\section{Clinical assessments}

Participants will be asked to complete a series of questionnaires within 20-90 min after each study drug administration. Although the wording of the validated pain questionnaires we will use during hospitalisation cannot 
be changed, we will aim to frame questions about pain intensity and interference with function in such a way as to avoid nocebo effects (ie, asking how well they are able to perform their daily activities, how well they are able to interact with visitors/family and how well they are sleeping instead of how much pain are they experiencing). The completion of a psychological battery and questionnaires unrelated to pain are optional.

- The following pain questionnaires will be administered each day of hospitalisation: pain intensity enjoyment of life and general activity (PEG) -3-item scale used to assess pain intensity and interference. ${ }^{23}$

- Emotional function using the specifiers from the recent International Classification of Diseases 11th Revision for pain ${ }^{24}$ and in accordance with the Initiative on Methods, Measurement, and Pain Assessment in Clinical Trials (IMMPACT) recommendations ${ }^{25}$

- Fear of Pain Questionnaire-widely used 30-item questionnaire to assess the fear of pain in clinical and nonclinical samples ${ }^{26}$

- Pain Catastrophizing Scale-widely used 13-item tool to measure catastrophic thinking related to pain ${ }^{27}$

- Pain Detect Questionnaire-7-item instrument to detect neuropathic pain components ${ }^{28}$

- Functional Pain Scale-Instrument to assess both subjective and objective components of pain ${ }^{29}$

The following Patient-Reported Outcomes Measurement Information System (PROMIS) short-form scales will be administered each day of hospitalisation ${ }^{30}$ :

- PROMIS Scale V.1.0-Pain Intensity 3a.

- PROMIS Short Form V.1.0—Pain Interference 8a.

- PROMIS Short Form V.1.1-Pain Behaviour 7a.

- PROMIS Short Form V.1.0-Prescription Pain Medication Misuse 7a.

- PROMIS Short Form V.1.0-Sleep Disturbance 8a.

- PROMIS Short Form V.1.0-Depression 8a.

- PROMIS Short Form V.1.0-Anxiety 8a.

\section{Phase Il: follow-up}

Post-discharge, participants will be monitored for subsequent use of opioids at each of the four study follow-up visits: 2 weeks, 1 month, 3 months and 6 months. If the participant has consented to optional blood samples, the participant will return for additional bloodwork during each follow-up visit. The research team will communicate with participants via calls, letters, emails or texts to inquire about their health and medication use. A HIPAAcompliant app-based platform (eg, MetricWire) will be used to continue administration of all questionnaires remotely and facilitate communication between participants and the study team during the follow-up period.

\section{Outcome measures}

The primary outcomes will be measured each day of hospitalisation including participant's pain intensity and improvement (12 self-reported measurements via questionnaires and scales; see Clinical Assessment section for pain-related questionnaires), the rate of opioid intake (frequency and dosage), the quantity and rate of opioid rescue intervention requests and the quantity of opioid rescue interventions declined. Opioid intakes and rescue interventions will be documented in Epic and the case report form (CRF) by clinicians and trained research coordinators.

Secondary outcomes will be used to measure the proportion of enrolled trauma participants relative to the number accrued and screened. This measurement will determine the feasibility and inform appropriate power for a larger randomised controlled trial in the future.

Exploratory outcomes will be measured during the follow-up visits within 6-month post-discharge: use of opioids including long-term prescriptions, psychological questionnaires and pain scales (see Clinical Assessment section).

\section{Patient and public involvement}

We conducted a survey of 50 participants suffering from chronic pain and a focus group in trauma inpatients to explore the possibility to conduct this dose-extending placebo-based trial. We have closely worked with the acute pain management team at STC to discuss feasibility and acceptability. Over several months of regular meetings and discussions to the original protocol, we worked diligently to ensure that the research question and outcome measures would be relevant to patients' priorities, experiences and preferences. Based on this feedback and preliminary work, modifications to the original protocol have been done to limit barriers and challenges.

\section{Sample size calculation}

This study will enrol a total of 159 participants with 53 participants assigned to each of the three arms. We predict in the FD group that patients will take a maximum of $90 \mathrm{mg}$ ( $5 \mathrm{mg}$ every 4 hours for 3 days) and in PR group that patients will take a maximum dose of $45 \mathrm{mg}(5 \mathrm{mg}$ every 4 hours for the first 24 hours and then estimated every 6 hours for 2 days) with an overall reduction of opioids in the hospitalisation window of approximately $50 \%$. We expect a small change in pain report $(0.25 \%)$, and account for $20 \%$ participant withdrawal. Therefore, we will need to recruit 159 participants, with 53 participants per group, to achieve a significant difference across groups (alpha set at 0.05 and $\mathrm{F}$ equal to 3.054).

\section{Data analysis}

To test $\mathrm{H} 1$ and H2, linear mixed model (LMM) that accounts for unbalanced and missing data ${ }^{31}$ will be used to test differences in the primary and secondary outcomes among the three groups controlling for sex, age, race as well as severity of baseline pain and duration of hospitalisation. The LMM will allow us to account for missing data. Data will be analysed using the Statistical Package for Social Sciences (SPSS) v.24 software.

\section{Data collection and quality management}

All source data collected via CRFs by research team members will be secured in the principal investigator's (PI) locked file cabinets located in the locked clinical 
studies suite at the School of Nursing, Floor 7. All electronic data will be kept on a password protected University of Maryland Baltimore (UMB) computer. All data files will be password protected and only accessible to the research team to ensure confidentiality.

Some or all data may be stored on web-based electronic databases, which are secure, HIPAA compliant software systems managed by the School of Medicine at the University of Maryland, Baltimore. The project files linked to this project on the databases will only be shared with study personal and most research study personal users will only have access to de-identified data. The Virtual Private Network (VPN) for the databases will ensure security of project data collected. We will maintain integrity via programme set-up such as valid values and data rules. The programme will also detect any missing data or errors to improve the quality of data.

\section{Data monitoring}

This study is reviewed annually by the PI and monitoring board. An interim analysis will be conducted when 25 participants have been randomised to each arm. Progress reports include reporting of adverse events, enrolment numbers, medical charts/clinical summaries, raw data, outcomes and preliminary analyses. The safety monitoring results will be reported to IRB.

\section{ETHICS AND DISSEMINATION}

All activities associated with this protocol are conducted in full compliance with current University of Maryland, Baltimore and University of Maryland, College Park's Human Research Protection Programs and IRB policies and procedures while maintaining compliance with federal regulations. This protocol is approved and is active with IRBs of both universities. Written informed consent is obtained from every participant.

\section{DISCUSSION}

Over the past 20 years in the USA, the prescription rates of opioids have increased by $300 \% .{ }^{4}$ Prescription rates are provoked by biased beliefs and lack of published comparative effectiveness research for the appropriate pace of opioid tapering for participants. ${ }^{32-35}$ Consequently, unnecessary opioid use post-contact with medical care remains persistent. In a previous study of approximately 36000 opioid naive participants with elective surgery, $6 \%$ of the participants used opioids persistently regardless of the type of surgery. ${ }^{36}$ Another study of trauma participants who were at-risk drinkers at the time of injury found that $7 \%-10 \%$ of the participants used prescription opioids 1 year later. ${ }^{37}$

This will the first study to assess feasibility and acceptability of dose-extending placebos to reduce opioid use in participants with acute trauma-related pain in a clinical setting. Dose-extending placebos induce effects through conditioning with an active drug. In our previously published analysis, we identified factors to consider prior to incorporating such use into clinical practice. ${ }^{12}$
Improvement in pain management is achieved with reduced amount of total drug intake in accordance with reinforcement learning principles. Extending the effects of a medication by interspersing placebos, rather than using only active medication for a treatment of equal duration, may reduce the overall intake of pain treatments. Although there is some risk of conditioned side effects (viz, the nocebo effect), the side effects associated with the medicine are likely to be reduced as well. ${ }^{12}$ In addition, in cases in which the active medication is habit-forming, dose-extending placebo use may decrease physiological or psychological dependence on medication. Furthermore, using dose-extending placebos for a portion of the treatment course, rather than using active medication for the entire course, can lower costs. Pharmacological conditioning has been used to study the mechanisms underlying placebo effects in the context of motor ${ }^{38}$ and endocrine ${ }^{39}$ systems in laboratory setting and real-world setting for treatment autoimmune diseases such as psoriasis ${ }^{40}$ and renal transplantation. ${ }^{41}$ Although dose-extending placebos can function as booster agents and trigger the action of active pain treatments in both animal and human research, there is a lack of research in acute pain clinical settings involving trauma patients. Pain patients undergoing elective surgery will be contemplated as an alternative to acute trauma patients if this protocol will be deemed unfeasible.

After careful review of ethical and clinical requirements, we designed a randomised, double-blinded, PoC clinical study. PoC studies are small-scale studies that test feasibility, acceptability, are generally designed to test whether a drug is active on a pathophysiologically relevant mechanism providing preliminary evidence of efficacy in a clinically relevant endpoint or demonstrate that some concepts have practical potential. Moreover, utilisation of app-based platforms dedicated to remote data collection during the follow-up period is useful for seamless glimpses into the patient's daily functioning. The increased convenience of remote data collection will also help retain participants throughout the study's duration.

The completion of this PoC study will help us to assess feasibility and acceptability opening up new research avenues that examine the learning mechanisms and doseextending placebos among participants to reduce opioid intakes in those patients with clinical acute pain due to traumatic injury and subsequent chronic pain.

\section{Author affiliations}

${ }^{1}$ Department of Pain and Translational Symptom Science, University of Maryland School of Nursing, Baltimore, Maryland, USA

${ }^{2}$ Department of Pharmaceutical Health Services Research, University of Maryland School of Pharmacy, Baltimore, Maryland, USA

${ }^{3} \mathrm{R}$ Adams Cowley Shock Trauma Center, University of Maryland Medical Center, Baltimore, Maryland, USA

${ }^{4}$ Investigational Drug Services, University of Maryland Medical Center, Baltimore, Maryland, USA

${ }^{5}$ Department of Anesthesiology, University of Maryland School of Medicine, Baltimore, Maryland, USA 
Acknowledgements Authors would like to acknowledge the following individuals for their contributions to the implementation: Jessica Rowe for helping with the Institutional Review Board and Jeffrey Gonzales for helping with the ideation of the manufacturing component.

Contributors LC, YF, TS, PC, SEL, CO, NH and SM designed the study; ML and LC took care of the strategies for the drug manufacturing and bedside delivery of the treatments; LC, SM, PC, RG, RSM and YF created the criteria of inclusion/exclusion; TS provided critical feedback on the manuscript; SY, NR, RSM, NH, CO and SEL designed the research tools from creating of the CRF to programming the scripts for text messaging the patients; $\mathrm{NH}, \mathrm{RSM}, \mathrm{RG}$ and $\mathrm{LC}$ ran the training of the nurses and physicians; SEL, NH and LC prepared the IRB protocol and ClinicalTrials.gov documents; SEL, CO and MNL drafted the first version of the manuscript; SEL, SM, TS, YF and LC completed the revision of the manuscript. All the authors reviewed and agreed with the latest version.

Funding This work was funded by the Agency for Healthcare Research and Quality (R24HS022135), MPowering the State: Strategic Partnership Grant (LC) to conduct clinical research related to new approaches to taper opioids for the management of acute pain.

Competing interests None declared.

Patient consent for publication Not required.

Provenance and peer review Not commissioned; externally peer reviewed.

Open access This is an open access article distributed in accordance with the Creative Commons Attribution Non Commercial (CC BY-NC 4.0) license, which permits others to distribute, remix, adapt, build upon this work non-commercially, and license their derivative works on different terms, provided the original work is properly cited, appropriate credit is given, any changes made indicated, and the use is non-commercial. See: http://creativecommons.org/licenses/by-nc/4.0/.

\section{REFERENCES}

1 Gaskin DJ, Richard P. The economic costs of pain in the United States. J Pain 2012;13:715-24.

2 Nahin RL. Estimates of pain prevalence and severity in adults: United States, 2012. J Pain 2015;16:769-80.

3 Sehgal N, Colson J, Smith HS. Chronic pain treatment with opioid analgesics: benefits versus harms of long-term therapy. Expert Rev Neurother 2013;13:1201-20.

4 Sullivan MD, Howe CQ. Opioid therapy for chronic pain in the United States: promises and perils. Pain 2013;154 Suppl 1:S94-100.

5 Zerzan JT, Morden NE, Soumerai S, et al. Trends and geographic variation of opiate medication use in state Medicaid fee-for-service programs, 1996 to 2002. Med Care 2006;44:1005-10.

6 Kroenke K, Krebs EE, Bair MJ. Pharmacotherapy of chronic pain: a synthesis of recommendations from systematic reviews. Gen Hosp Psychiatry 2009;31:206-19.

7 Florence CS, Zhou C, Luo F, et al. The economic burden of prescription opioid overdose, abuse, and dependence in the United States, 2013. Med Care 2016;54:901-6.

8 Chou R. Long-Acting opioids for chronic noncancer pain were linked to mortality. Ann Intern Med 2016;165:JC34.

9 Chou R, Turner JA, Devine EB, et al. The effectiveness and risks of long-term opioid therapy for chronic pain: a systematic review for a national Institutes of health pathways to prevention workshop. Ann Intern Med 2015;162:276-86.

10 Volkow ND, McLellan AT. Opioid Abuse in Chronic Pain-Misconceptions and Mitigation Strategies. N Engl J Med 2016;374:1253-63.

11 Kaplovitch E, Gomes T, Camacho X, et al. Sex differences in dose escalation and overdose death during chronic opioid therapy: a population-based cohort study. PLoS One 2015;10:e0134550.

12 Colloca L, Enck P, DeGrazia D. Relieving pain using dose-extending placebos: a scoping review. Pain 2016;157:1590-8.

13 Colloca L, Miller FG. Harnessing the placebo effect: the need for translational research. Philos Trans $R$ Soc Lond B Biol Sci 2011;366:1922-30.

14 Colloca L. The placebo effect in pain therapies. Annu Rev Pharmacol Toxicol 2019;59:191-211.

15 Klinger R, Colloca L, Bingel U, et al. Placebo analgesia: clinical applications. Pain 2014;155:1055-8.
16 Colloca L, Benedetti F. How prior experience shapes placebo analgesia. Pain 2006;124:126-33.

17 Klinger R, Soost S, Flor $\mathrm{H}$, et al. Classical conditioning and expectancy in placebo hypoalgesia: a randomized controlled study in patients with atopic dermatitis and persons with healthy skin. Pain 2007;128:31-9.

18 Fiorio M, Recchia S, Corrà F, et al. Behavioral and neurophysiological investigation of the influence of verbal suggestion on tactile perception. Neuroscience 2014;258:332-9.

19 Fiorio M, Recchia S, Corrà F, et al. Enhancing non-noxious perception: behavioural and neurophysiological correlates of a placebo-like manipulation. Neuroscience 2012;217:96-104.

20 Colloca L, Howick J. Placebos without deception: outcomes, mechanisms, and ethics. Int Rev Neurobiol 2018;138:219-40.

21 Brown R, Deyo B, Riley C, et al. Screening in trauma for opioid misuse prevention (STOMP): study protocol for the development of an opioid risk screening tool for victims of injury. Addict Sci Clin Pract 2017;12

22 Webster LR, Webster RM. Predicting aberrant behaviors in opioidtreated patients: preliminary validation of the opioid risk tool. Pain Med 2005;6:432-42.

23 Krebs EE, Lorenz KA, Bair MJ, et al. Development and initial validation of the PEG, a three-item scale assessing pain intensity and interference. J Gen Intern Med 2009;24:733-8.

24 Treede R-D, Rief W, Barke A, et al. Chronic pain as a symptom or a disease: the IASP classification of chronic pain for the International classification of diseases (ICD-11). Pain 2019;160:19-27.

25 Cooper SA, Desjardins PJ, Turk DC, et al. Research design considerations for single-dose analgesic clinical trials in acute pain: IMMPACT recommendations. Pain 2016;157:288-301.

26 Asmundson GJG, Bovell CV, Carleton RN, et al. The fear of pain Questionnaire-Short form (FPQ-SF): factorial validity and psychometric properties. Pain 2008;134:51-8.

27 Darnall BD, Sturgeon JA, Cook KF, et al. Development and validation of a daily pain Catastrophizing scale. J Pain 2017;18:1139-49.

28 Freynhagen R, Baron R, Gockel U, et al. painDETECT: a new screening questionnaire to identify neuropathic components in patients with back pain. Curr Med Res Opin 2006;22:1911-20.

29 Gloth FM, Scheve AA, Stober CV, et al. The functional pain scale: reliability, validity, and responsiveness in an elderly population. J Am Med Dir Assoc 2001;2:110-4. 3rd.

30 Cella D, Riley W, Stone A, et al. The patient-reported outcomes measurement information system (PROMIS) developed and tested its first wave of adult self-reported health outcome item banks: 2005 2008. J Clin Epidemiol 2010;63:1179-94.

31 Ringham BM, Kreidler SM, Muller KE, et al. Multivariate test power approximations for balanced linear mixed models in studies with missing data. Stat Med 2016;35:2921-37.

32 Berna C, Kulich RJ, Rathmell JP. Tapering long-term opioid therapy in chronic noncancer pain: evidence and recommendations for everyday practice. Mayo Clin Proc 2015;90:828-42.

33 Frank JW, Levy C, Matlock DD, et al. Patients' perspectives on tapering of chronic opioid therapy: a qualitative study. Pain Med 2016;17:1838-47.

34 McAuliffe WE. Prescribing opioids for chronic pain. JAMA 2016;316.

35 Wen LS, Lloyd MC. Prescribing opioids for chronic pain. JAMA 2016;316.

36 Brummett CM, Waljee JF, Goesling J, et al. New persistent opioid use after minor and major surgical procedures in US adults. JAMA Surg 2017:152:e170504

37 Field CA, Cochran G, Caetano R, et al. Postdischarge nonmedical use of prescription opioids in at-risk drinkers admitted to urban level I trauma centers. J Trauma Acute Care Surg 2014;76:833-9.

38 Benedetti F, Colloca L, Torre E, et al. Placebo-responsive Parkinson patients show decreased activity in single neurons of subthalamic nucleus. Nat Neurosci 2004;7:587-8.

39 Benedetti F, Pollo A, Lopiano L, et al. Conscious expectation and unconscious conditioning in analgesic, motor, and hormonal placebo/nocebo responses. J Neurosci 2003;23:4315-23.

40 Ader R, Mercurio MG, Walton J, et al. Conditioned pharmacotherapeutic effects: a preliminary study. Psychosom Med 2010;72:192-7.

41 Kirchhof J, Petrakova L, Brinkhoff A, et al. Learned immunosuppressive placebo responses in renal transplant patients. Proc Natl Acad Sci U S A 2018;115:4223-7. 